\title{
Facile and Large-Scale Production of ZnO/Zn-Al Layered Double Hydroxide Hierarchical Heterostructures
}

\author{
Jinping Liu, ${ }^{* \dagger}$ Xintang Huang, ${ }^{* \dagger}$ Yuanyuan Li, ${ }^{\dagger}$ K. M. Sulieman, ${ }^{\dagger}$ Xiang He, ${ }^{\dagger \dagger}$ Fenglou Sun ${ }^{\dagger \dagger}$ \\ Department of Physics, Central China Normal University, Wuhan 430079, P. R. China, and \\ Plasma Institute, South-Central University for Nationalities, Wuhan 430074, P.R. China
}

Figure S1. A typical SEM image of the ZnO/Zn-Al LDH hierarchical heterostructures dispersing sparsely. From this picture, underdeveloped nanostructures can be observed. Furthermore, we can easily find that $\mathrm{ZnO}$ nanorods just grow at the edges of 2D hexagonal platelets.

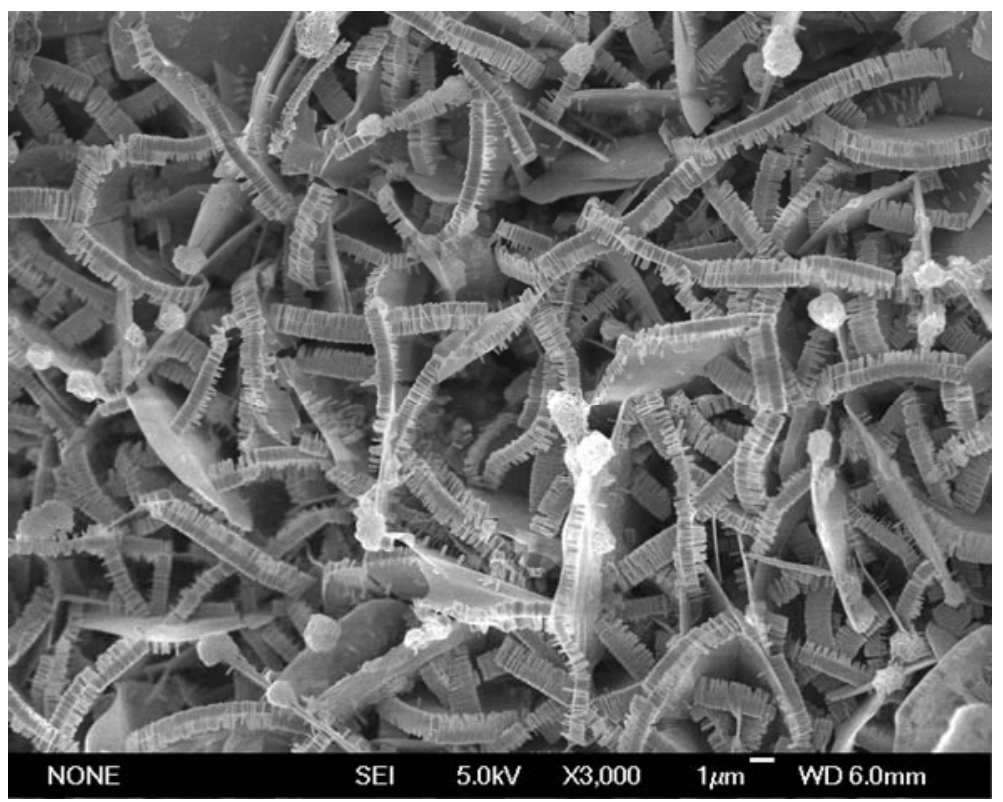

\footnotetext{
* To whom correspondence should be addressed: Fax +86-027-67861185; E-mail: xthuang@phy.ccnu.edu.cn (X. Huang); ljpphyccnu@mails.ccnu.edu.cn (J. Liu).

${ }^{\dagger}$ Central China Normal University.

${ }^{\dagger \dagger}$ South-Central University for Nationalities.
} 
Figure S2. EDS result of the $\mathrm{ZnO} / \mathrm{Zn}-\mathrm{Al} \mathrm{LDH}$ heterostructures. The carbon element is from $\mathrm{Zn}-\mathrm{Al}$ LDHs $\left(\mathrm{Zn}_{6} \mathrm{Al}_{2}(\mathrm{OH})_{16} \mathrm{CO}_{3} \cdot \mathrm{nH}_{2} \mathrm{O}\right)$. The presence of Pt is due to charging of the sample.

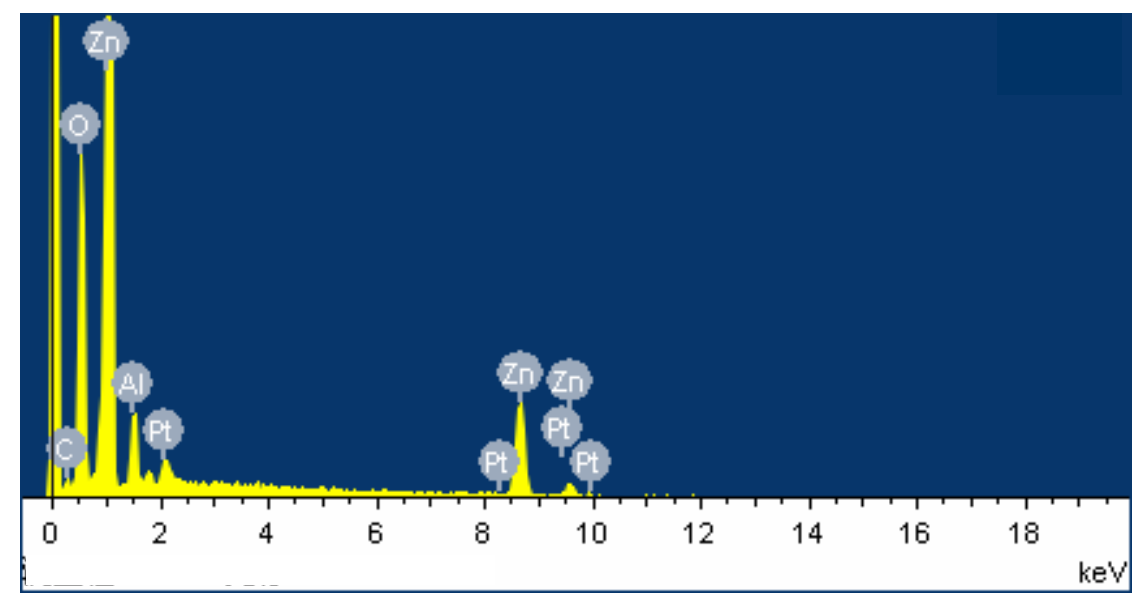

Figure S3. HRTEM image of the tip of an individual $\mathrm{ZnO}$ nanorod.

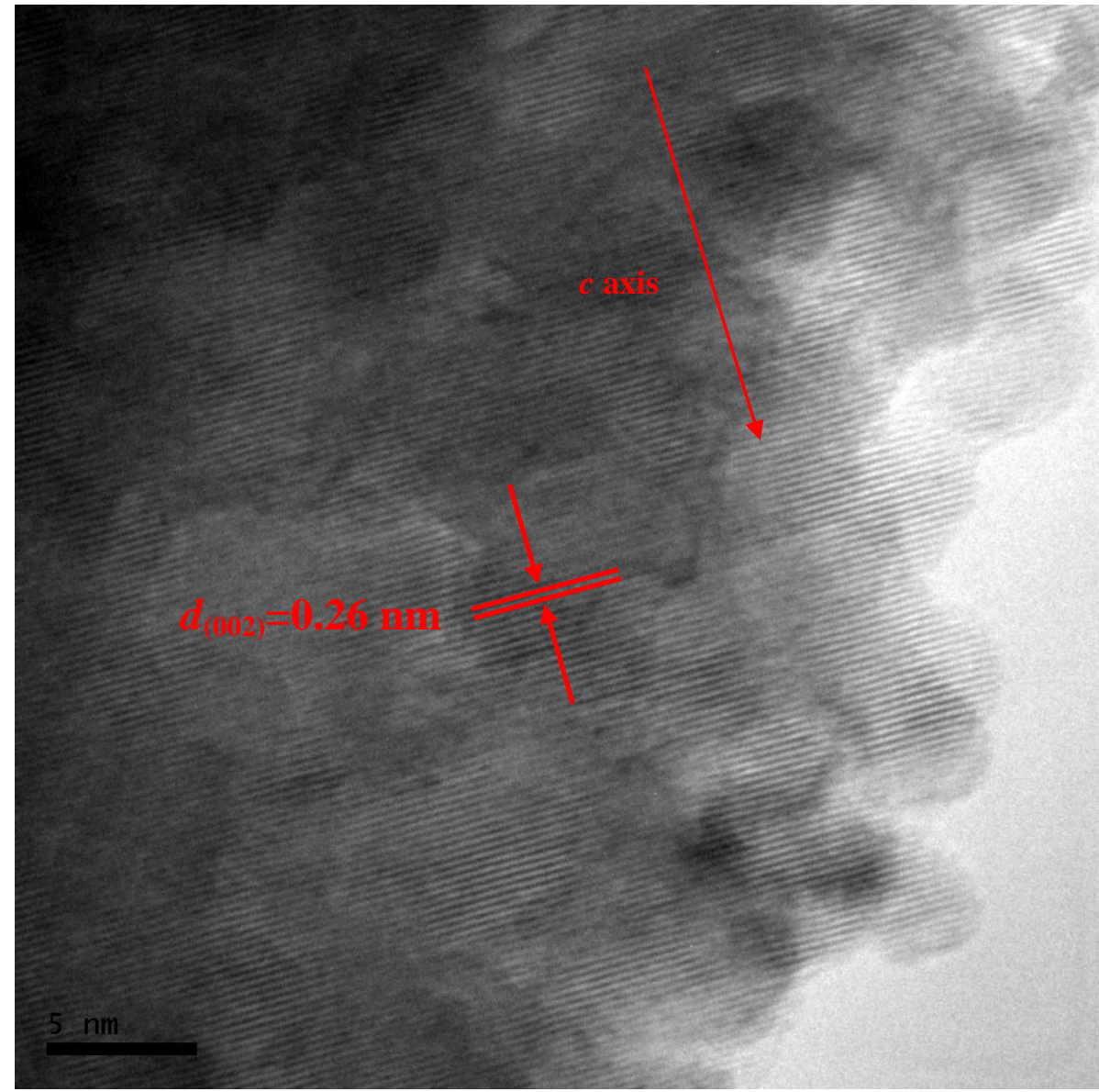


Figure S4. Low-magnification SEM image of densely-packed ZnO nanorods.

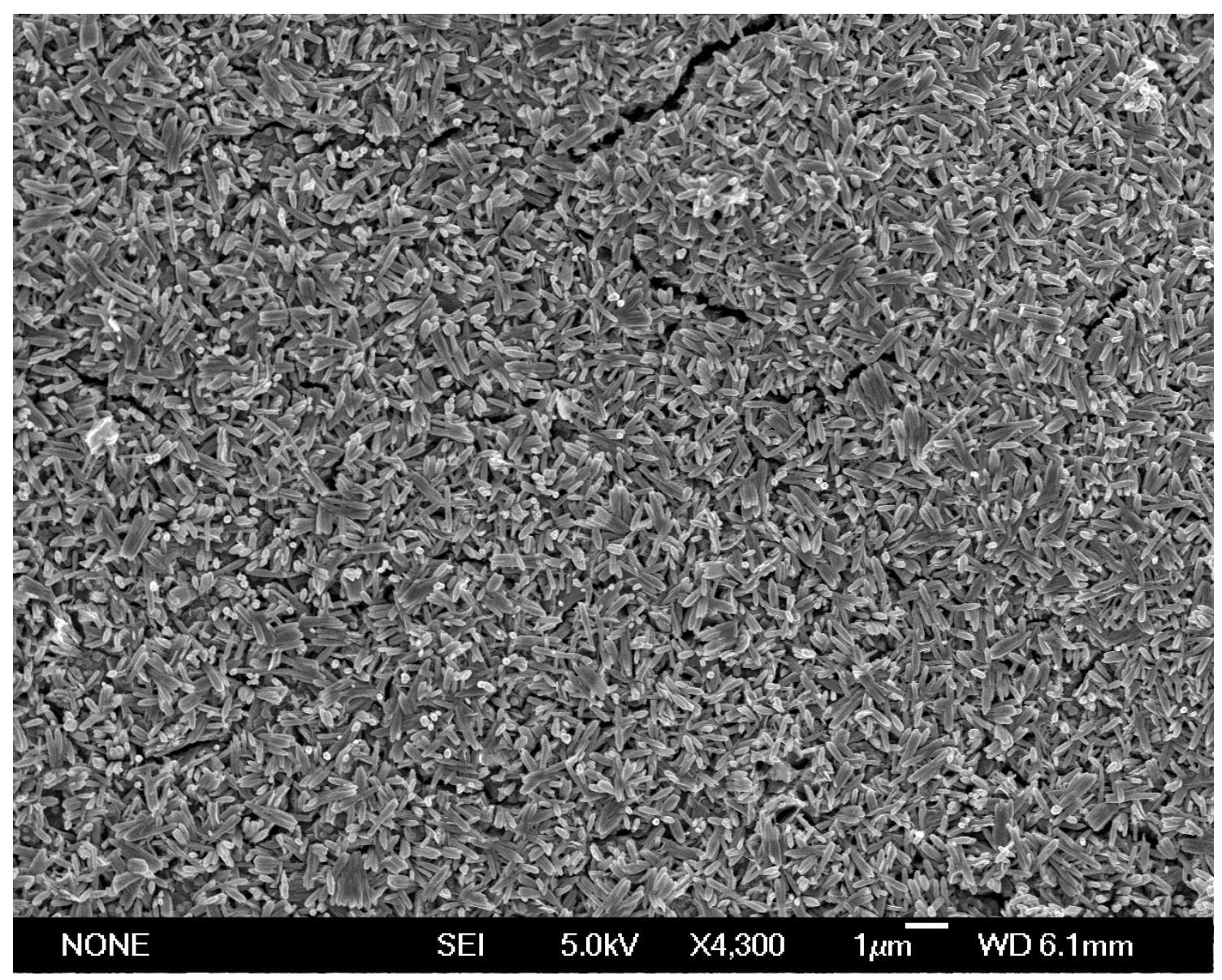

Figure S5. EDS result of the densely-packed ZnO on the Zn-Al LDHs. Because of the small quantity of Zn-Al LDHs, signals from carbon (C) element cannot be detected, which is different from the result shown in Figure S2.

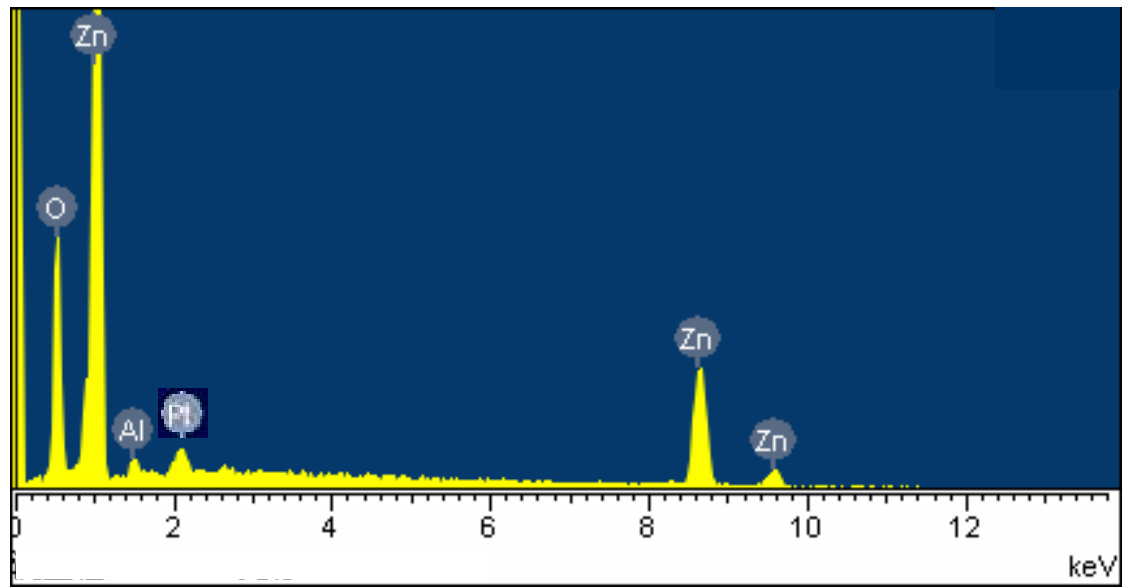

